\title{
MuTent: Dynamic Android Intent Protection with Ownership-Based Key Distribution and Security Contracts
}

\author{
Pradeep Kumar D S ${ }^{1}$, Jaejong Baek ${ }^{2}$, Tiffany Bao $^{2}$, Yan Shoshitaishvili ${ }^{2}$, Adam L Doupe ${ }^{2}$, \\ Ruoyu Wang ${ }^{2}$, and Gail-Joon $\mathrm{Ahn}^{2}$ \\ Arizona State University \\ ${ }^{1}$ \{pradeepkumarst\}@ gmail.com \\ ${ }^{2}\{$ jbaek7, tbao, yans, doupe, fishw, gahn $\} @$ asu.edu
}

\begin{abstract}
Intents are the plain-text based message object used for ICC by the Android framework. Hence the framework essentially lacks an inbuilt security mechanism to protect the visibility, accessibility, and integrity of Intent's data that facilitates adversaries to intercept or manipulate the data.

In this work, we investigate the Intent protection mechanism and propose a security-enhanced Intent library $\mu$ Tent that allows Android apps to securely exchange sensitive data during ICC. Differently from the existing mechanism, $\mu$ Tent provides accessibility and visibility of Intent data by validating the receiver's capability and provides integrity by using encryption and the Arc security contract code. Especially, ICC is initiated by exchanging $\mu$ Tent and follows a novel ownership-based key distribution model, that restricts the malware apps without permission from deciphering data. Through the evaluation, we show that $\mu$ Tent can improve the security for popular Android apps with minimal performance overheads, demonstrated using F-Droid apps.
\end{abstract}

\section{Introduction}

Intents are messaging object in the Android platform used to coordinate an activity, start a service, or as payload carrying sensitive data, operations, and actions to be performed by the target component (for example, the BroadcastReceiver or IntentService). Utilizing Intents without well-defined security mechanism inevitably exposes the system to threats like backdoors, which attackers can use to intercept sensitive information. For example, CVE-2018-9489 [1] reports an unintentional information leak, where the Android's WiFi module leaks the MAC address of the device to all the registered component's without validating the receiver's permissions.
There are mechanisms to prevent a malware component from receiving the Intent data. These mechanisms prevent Inter-Component Communication (ICC) attacks by statically analyzing the DEX files and dynamically monitoring to detect security vulnerabilities [2, 3]. SALMA [4] builds a Multiple-Domain-Matrix (MDM), and incrementally analyzes the App security in response to incremental system changes. Barros et al. [5] present a static program analysis where the developer can annotate the Intent data with security levels, thereby restricting data flow across incompatible source and sink.

With these filtering techniques, we can protect Intent flow across apps, however, protecting Intents dynamically from content pollution and information confidentiality through encryption of data have not been fully covered for practical use. For example, an intermediate component may pollute the Intent data by direct mutation or through reflection, thereby all the information that is used and forwarded by that subsequent component will be corrupted. The result is an epidemic propagation of a corrupted Intent which will be multiplied upon further propagation.

In terms of the confidentiality issue, EventGuard [6] proposes a mechanism to guard the information in a pub-sub environment through encryption. It uses a meta-service layer that connects the subscriber and publisher based on the filter. However, EventGuard treats every subscriber as equal with the same capability. This may not be applicable for Android, since in Android every app (subscriber) should be treated differently based on the capability of application (i.e. security properties defined in their AndroidManifest.xml and acquired at runtime by user granting/revoking permissions). Thus, any changes in App's permissions make an impact on the capability of corresponding App's component's. Overall, the lack of a holistic security framework having both integrity and confidentiality is a primary challenge in 
Table 1: Types of Android Intent Attack. [Note: $\mathrm{T}_{\mathrm{r}}=$ Benign; $\mathrm{M}_{\mathrm{r}}=$ Malware; $\mathrm{A}_{\mathrm{a}}=$ Active Attack; $\mathrm{P}_{\mathrm{a}}=$ Passive Attack; $\mathrm{C}_{\mathrm{b}}=$ Channel Based; $\mathrm{I}_{\mathrm{b}}=$ Intent Based $]$

\begin{tabular}{lllll}
\hline & Receiver's & Type & \multicolumn{1}{c}{ Mode } & $\begin{array}{c}\text { Vulnerability } \\
\text { Type }\end{array}$ \\
\hline UIR & $M_{r}$ & Leak & $\mathrm{P}_{\mathrm{a}}$ & $\left(\mathrm{C}_{\mathrm{b}}, \mathrm{I}_{\mathrm{b}}\right)$ \\
PE & $\mathrm{M}_{\mathrm{r}}$ & Leak & $\left(\mathrm{A}_{\mathrm{a}}, \mathrm{P}_{\mathrm{a}}\right)$ & $\left(\mathrm{C}_{\mathrm{b}}, \mathrm{I}_{\mathrm{b}}\right)$ \\
$\mathrm{IM}$ & $\mathrm{T}_{\mathrm{r}}$ or $\mathrm{M}_{\mathrm{r}}$ & Mutation & $\mathrm{A}_{\mathrm{a}}$ & $\left({ }_{-}, \mathrm{I}_{\mathrm{b}}\right)$ \\
\hline
\end{tabular}

Android system to protect the Intents during ICC. In this paper, we propose $\mu$ Tent, a secure and pluggable Intent library supporting encryption of Intent data, an ownership-based key generation and distribution mechanism, and dynamic verification of receiver's capability and access privileges.

$\mu$ Tent has three enhanced security features: (1) dynamically verifying the sender and receiver capabilities for subset property, i.e. the source (sender or publisher) component's capability must have a subset relationship with the sink (receiver or subscriber) component's capability; (2) $\mu$ Tents carry security contract code written in our own domain specific language called Arc explained in 53.3 that will be verified dynamically before granting/revoking access to the receiver; (3) $\mu$ Tents are encrypted and the key is withheld by the $\mu$ Tent's owner, receiver component must pass conditions (1) and (2) to receive the key.

In summary, $\mu$ Tent provides the following advantages: firstly, capability-based access guarantees that $\mu$ Tent is secure wherever it goes; secondly, the Arc contracts (called simply as contract) can control not only the legal information flow in ICC but also the legal operations such as accessibility and visibility that are allowed on the Intent data; finally, every $\mu$ Tent object is dynamically encrypted by its owner, and makes any access satisfy the above two conditions to fetch the decryption key from $\mu$ Tent's owner. To the best of our knowledge, $\mu$ Tent is the first holistic security framework that handles dynamic Intent mutation and information confidentiality.

As a proof of our mechanism, we have implemented $\mu$ Tent as a pluggable Java Library, which can be used by developer's at the application layer by replacing Android's Intent library.

\section{Overview}

Intent-based communication may not trust the publishers and subscribers, and may not trust the transit path as well. A study of 7,406 applications by Ali Feizollah et.al. [7] found that $91 \%$ of the applications are using Android Intents to communicate

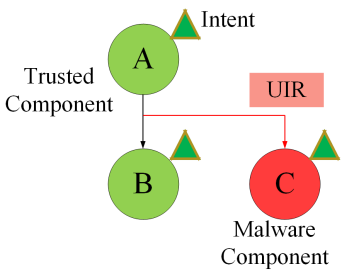

(a)

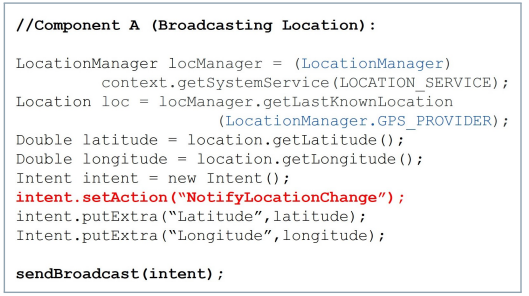

(b) Code.

Figure 1: Unauthorized Intent Receipt Attack.

across components, among which $28.78 \%$ are implicit Intents, where the receiver component is decided by the Android framework, thereby creating a potential risk of leaking information to malicious components, and $71.22 \%$ apps uses explicit Intents that may introduce mutation attacks where the receiver component can mutate the Intent data consciously or fortuitously.

We present three types of common Intent-based attacks (see Table 1), that actively or passively confronts the Intent data security, two Intent leak attacks and one Intent mutation attack that impair the confidentiality and privacy of Android's ICC. Unauthorized Intent Receipt (UIR) and Privilege Escalation (PE) are the passive attack model where the malicious receiver $\left(M_{r}\right)$ remains stealthy sneaking Intent information directly or indirectly from the source. For example, the Android WiFi module (tested on AOSP v8.1.0_r70) leaks the device sensitive information such as (the WiFi network name, BSSID, local IP addresses, DNS server information and the MAC address) to all registered applications running on that user device irrespective of the application's capability (permissions declared in AndroidManifest.xml and actual permissions granted by the user) (CVE-2018-9489 [1]). In privilege escalation, a malware component acquires sensitive information indirectly through a privilege component by using its loophole. In active mutation attack, the attacker $\left(T_{r}\right)$ intercepts the transit Intent data and modifies the information that affects the behavior of the components further.

\subsection{Motivating Example}

We demonstrate our examples with the following four components: benign component A (acts as source) 


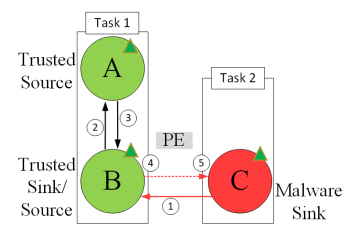

(a)

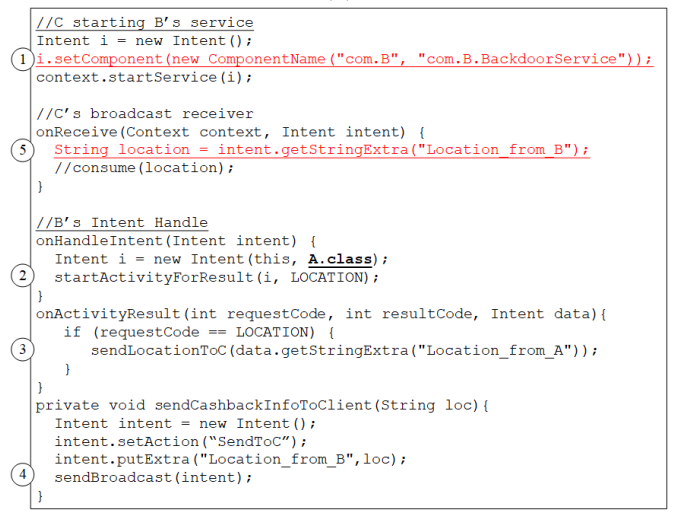

(b) Code.

Figure 2: Privilege Escalation Attack.

with capability $\mathrm{C}_{\mathrm{a}}=($ LOCATION, READ_CONTACTS $)$, a crafted component $B$ with capability $C_{b}=($ LOCATION, READ_CONTACTS), that can act either as benign (2.1.1) or as malware 2.1.2, the malware component $\mathrm{C}$ with capability $C_{c}=($ INTERNET $)$, and the benign component $\mathrm{D}$ with capability $\mathrm{C}_{\mathrm{d}}=($ LOCATION, READ_CONTACTS $)$.

2.1.1. Intent Leak Attacks. UIR - As shown in Figure 11 A broadcasts the Intent with sensitive information (i.e., device locations) to B, (Figure 1b), using action ("NotifyLocationChange"). Now by registering to the action ("NotifyLocationChange") the malware component $\mathrm{C}$ sniffs the transit Intent from $\mathrm{A}$ (shown in Figure 1a), thereby gaining access to the sensitive information.

PE - In this case $\mathrm{C}$ without location permission indirectly accesses the location data through the benign B (Figure 2b (1), (2)). B receives the location information from A (Figure 2b (3) and broadcasts it to the $\mathrm{C}$ (Figure 2b (4), (5)).

2.1.2. Mutation Attack. This is a type of inside attack, where the receiver is a trusted component performing an active attack on the intent by modifying the data consciously or fortuitously and passing to other components further. We demonstrate a similar example in Figure 3a. (1) A receives the following details sender and receiver account numbers, amount, and bank code, (2) B validates the sender and receiver account numbers, bank code, and the amount before initiating sending process, and (3) D processes the money transfer

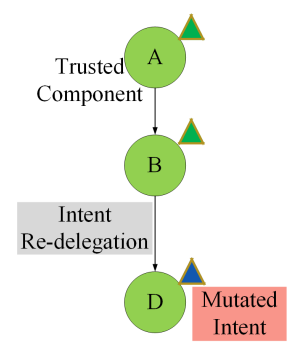

(a)

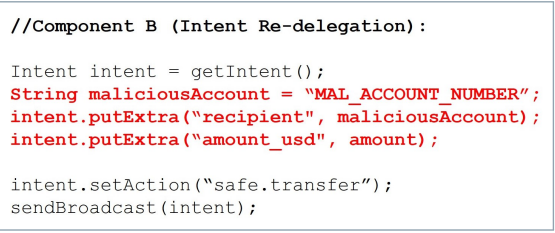

(b) Code.

Figure 3: Intent Mutation Attack.

from sender to receiver account. Here, B is performing mutation-attack by modifying the account number and the amount using the hard-coded values or inputs taken from other side-channels [8], Figure 3b] Now D processes money transfer on the mutated data. This type of attack introduces unpredictable security issues that are difficult to capture using application capability or static analysis. Failure of data integrity and data security creates attacks such as man-in-the-middle attacks [9, 10] where the attacker can modify the transit data that may lead to a catastrophe in the banking applications.

2.1.3. Challenges. The Intent flow defines the source component from where the Intent originates and the sink component of Intent. Android initiates ICC by creating the Intent inside the receiver context. Since we focus on providing Intent-based ICC filtering, it becomes challenging to restrict ICC from Intents before creating Intent inside the receiver's context. Once Intent is created within receiver's context the receiver obtains complete control over the Intent object by gaining access the Intent object through direct API or through reflection, i.e., data once copied into the memory of an untrusted application can be exported thereby failed to achieve data secrecy [11].

The channel-based attacks are handled by filtering the receiver's through capability subset mechanism $(\$ 3.4)$, i.e. source's components capability $\left(C_{s}\right)$ should be subset of the subscriber's capability $\left(C_{r}\right)$ (i.e. $C_{s} \subseteq$ $\left.C_{r}\right)$ ). This approach ensures the confidentiality and accessibility, however the guarantee for integrity is not addressed here.

On the other hand, the Intent-based mutation attack happens even if the receiver is benign. This problem requires limiting the receiver from performing 


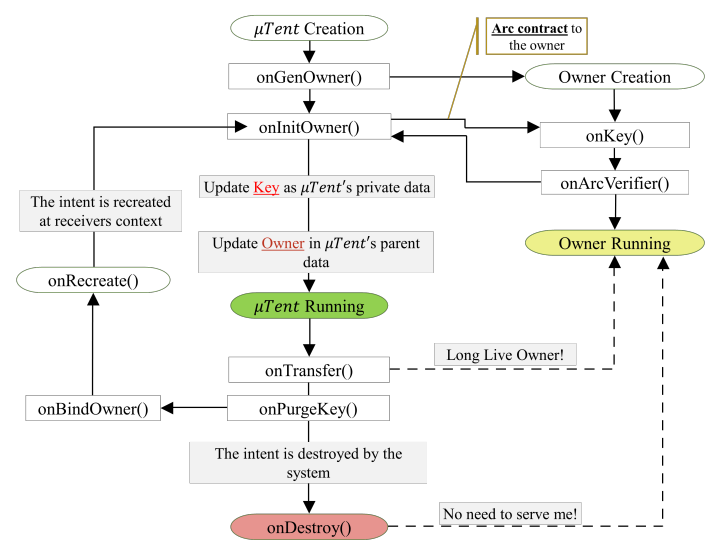

Figure 4: $\mu$ Tent Life Cycle.

malicious operations on the Intent data like mutating the information.

To this end, we identified three major challenges in protection against Intent-based attacks to ensure confidentiality, accessibility and integrity: encrypting the Intent object so that the malicious receiver cannot eavesdrop the Intent bypassing the validation process, (2) dynamically validating the capability within the receiver's context, (3) limiting mutation on Intent data.

2.1.4. Approach and Assumptions. UIR in Figure 1 and PE in Figure 2, the component A (publisher) has a capability subset relationship with $\mathrm{B}$ (the subscriber) (i.e. $C_{a} \subseteq C_{b}$ ), therefore, $B$ being the superset of $A$ has all capabilities that $A$ has and hence we recognize $B$ as benign. Being benign $B$ can access the Intent data from A, although the operations on Intent data (i.e. mutation) are not guaranteed. On the other hand, the component $\mathrm{C}$ does not have superset relationship with component A or B (i.e. $\left.\left(C_{a} \vee C_{b}\right) \nsubseteq C_{c}\right)$. Therefore component $\mathrm{C}$ cannot even access the Intent, thus we recognize $\mathrm{C}$ as malware.

The Intent-based mutation attack requires the developer to send a runtime Arc contract along with the Intent specifying its mutable or immutable nature during the transit. The Arc contract imposes the permitted operations and visibility restrictions of the receiver within the receiver's context. The contract is added during Intent creation and cannot be modified later.

Currently, our model has limits to handle the following types of leaks: 1) log leaks - leaking the data extracted from the Intent object to the log channels; 2) creating a new Intent object by copying the data from the original Intent and distributing it further along the transit path. $\mu$ Tent protects the Intent at object level using encryption and dynamic contracts rather than at the object's data level.

\section{3. $\mu$ Tent}

In this section, we present the design and architecture of $\mu$ Tent. $\mu$ Tent is a secure and pluggable Intent library supporting encryption of Intent data with an ownership-based key generation and distribution mechanism and dynamic verification of receiver's capability and access privileges. $\mu$ Tent encrypts the extras and data attributes, however, does not encrypt action, category, type, component that are used by Android framework for ICC, and the owner's Unique Resource Identifier (URI) and Arc contract.

\section{1. $\mu$ Tent Primitives}

Each $\mu$ Tent has two parts, (1) the $\mu$ Tent owner (for encryption key generation and distribution, and receiver capability validation) and (2) the Arc contract defines additional access restrictions/capabilities imposed on the receiver. The owner [12] provides a logical encapsulation boundary to the $\mu$ Tent object. Before initializing the $\mu$ Tent at the receiver's context, the receiver's context should be authorized by the $\mu$ Tent's owner by validating the receiver's capability and the Arc contract (if given).

Formally, the relationship between $\mu$ Tent and its corresponding owner $(\mathrm{O})$ can be represented as $\mu$ Tent $\preceq$ O. The symbol $\preceq$ is known as within (or dominated by) relationship, says that the owner (O) encapsulates the $\mu$ Tent with a capability-based logical boundary, protecting $\mu$ Tent from any unauthorized access from outside the ownership boundary. Thereby, all access to the $\mu$ Tent must pass through its corresponding owner $(\mathrm{O})$. This ownership property ensures that possession of a $\mu$ Tent object by a receiver does not imply permission to access the information of that $\mu$ Tent. Additionally, the Arc contracts control's the legal operations allowed on the Intent data.

\section{2. $\mu$ Tent Life Cycle}

ICC initiates $\mu$ Tent's lifecycle by exchanging $\mu$ Tent across component's. Figure 4 shows various states in the $\mu$ Tent's lifecycle from the creation to the destruction of the $\mu$ Tent.

3.2.1. Creating $\mu$ Tent Owner. The pseudo code is given in Algorithm1 It takes the $\mu$ Tent current context, the Arc contract, and the current timestamp from the environment. First the creation process verifies the correctness of the Arc version (mismatch in version throws an exception). Then the owner's unique ID is generated UUID, and the timestamp $\left(t_{s}\right)$. The owner 


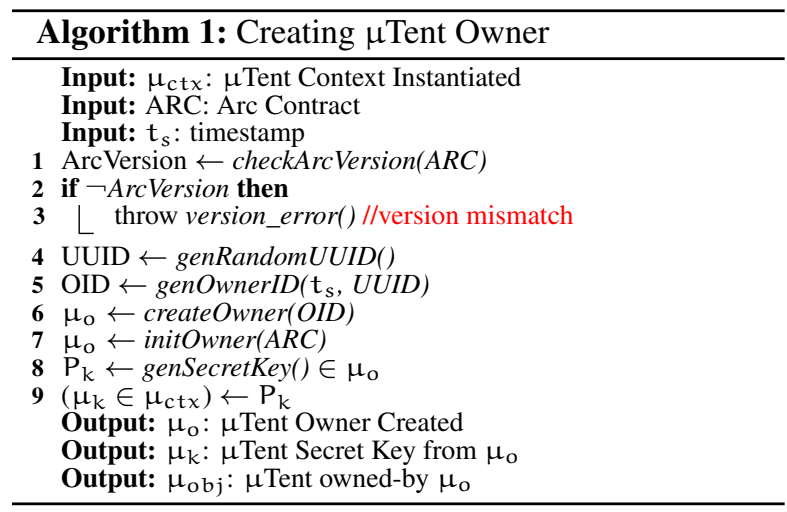

then initiate the call to generate a secret key $\left(P_{k}\right)$. The generated key $\left(P_{k}\right)$ is stored into the owner $\mu_{0}$.

3.2.2. Accessing $\mu$ Tent Object. The pseudo code is given in Algorithm 2 When $\mu$ Tent receives request for get/set methods of Intent data, the $\mu$ Tent validates the current context capability and privileges. On successful verification, the $\mu$ Tent will request the owner for the secret key $\left(P_{k}\right)$. This shared secret key is used by the $\mu$ Tent to decrypt the Intent data.

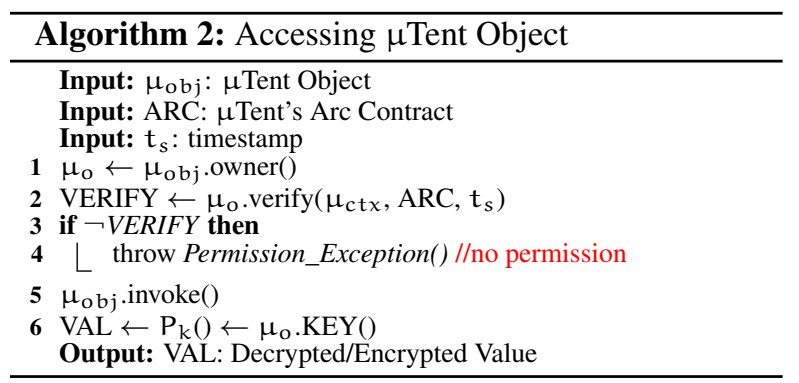

3.2.3. Migrating $\mu$ Tent Object. The pseudo code is given in Algorithm 3. Migration process gets initiated during ICC: startActivity, sendBroadcast, startService, startActivityForResult, etc. Migration consists of three steps: (1) the $\mu$ Tent sends a signal to its corresponding owner to wait till it validates the receiver's context before recreation. (2) The $\mu$ Tent's data will be encrypted with the secret key, (3) The secret key is purged from the $\mu_{\mathrm{obj}}$, (4) followed that, the owner ID is added to $\mu_{\mathrm{obj}}$, (5) finally, $\mu_{\mathrm{obj}}$ is parceled and migrated from the current context to the receiver's context.

3.2.4. Recreating $\mu$ Tent Object. The pseudo code is given in Algorithm 4 . Inside the receiver's context, first the $\mu$ Tent communicates to its source owner, by sending the Arc contract, receiver's capability and time stamp. Upon successful verification, the owner will send the secret key to the $\mu$ Tent. On verification failure, the $\mu$ Tent will not get its decryption key.

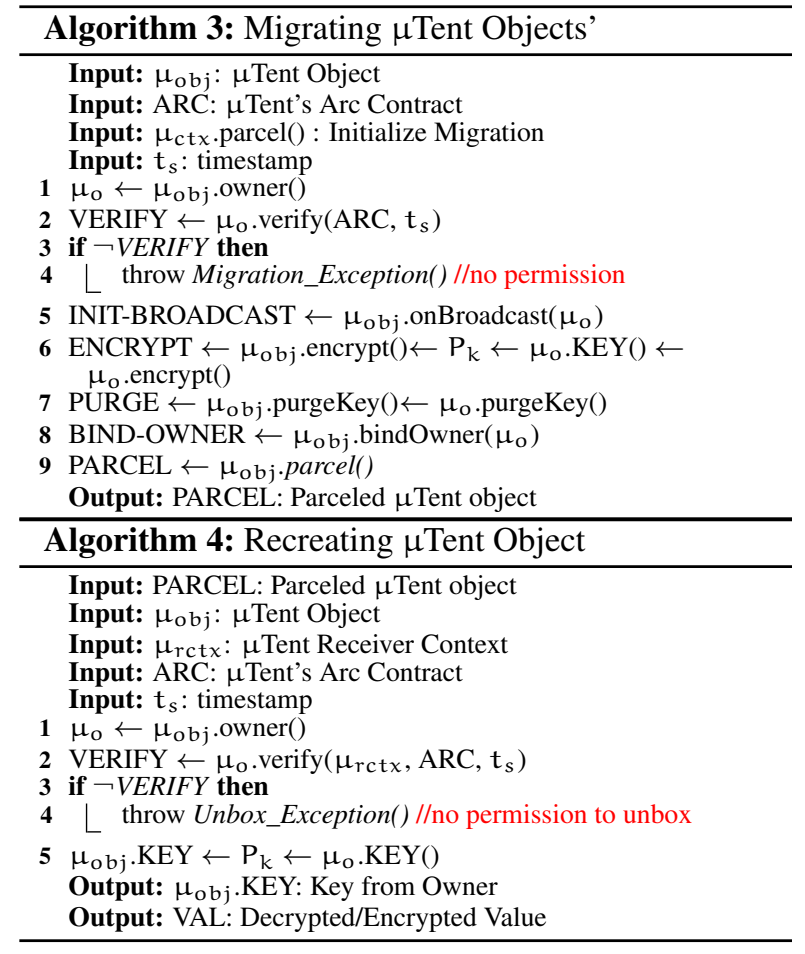

3.2.5. Destroying $\mu$ Tent Object. $\mu$ Tent destruction state pseudo code is given in Algorithm 5. Here, the $\mu$ Tent sends an notify signal to its owner. Followed by, purging the secret key, and $\mu$ Tent's destroy signal to its corresponding owner. The destroy signal removes the $\mu$ Tent's dependency from its owner, and now the owner can self-destruct itself.

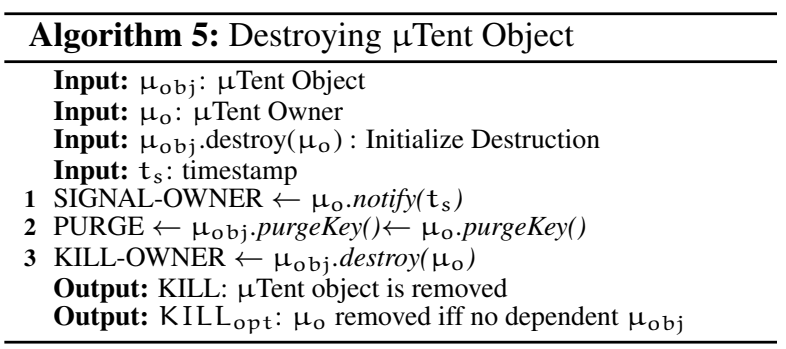

\subsection{Arc Language Specifications}

The Arc is a design by contract language, with basic constructs for Android security. The Arc follows annotation such as syntax similar to JML [13]. The current Arc implementation supports eight basic constructs: (1)@sameProcess; (2)@sameTask; (3) @read; (4) @write; (5) ^; (6) $\vee ;(7) \neg ;(8) \stackrel{\text { impl }}{\longrightarrow}$.

The Arc grammar is given in Figure 5 Constraints describe the receiver's context, and the access keyword 
is used to specify the accessibility. The meta variable $\mathbf{P}$ range over the contracts. The meta variable $\mathbf{O P}$ describe the logical operators.

@sameProcess: It limits $\mu$ Tent's visibility and accessibility within the same process (source) that created the $\mu$ Tent, thereby component's from different processes cannot access the $\mu$ Tent information. By default, $\mu$ Tent is accessible across processes.

@sameTask: It ensures that the receiver's task ID and task affinity (in case of BroadcastReceiver and IntentService) is same as the source's task ID and task affinity. By default, $\mu$ Tent is accessible across task.

@read: It protects the $\mu$ Tent data from any future (unintended) modifications. Negation of read specifies that the $\mu$ Tent cannot be read by the receiver. For example the Arc contract " $\neg @$ sameTask $\stackrel{\text { impl }}{\longrightarrow} \neg @$ read" specifies no read constraint to the receiver's' belongs to different task. That is receiver's from different task cannot read the data. Under this condition the receiver can only forward/drop the $\mu$ Tent and cannot consume any information from it as they are protected with encryption.

@write: It enables $\mu$ Tent data to get modified, by default $\mu$ Tent is writable. Negation of @write (i.e. $\neg @$ write) means that the $\mu$ Tent is read-only.

$\wedge I \vee I \neg:$ The logical represents general math logic for AND | OR I NOT operation respectively.

$\stackrel{\text { impl }}{\longrightarrow}$ : The implication operation can be expressed as $(\mathbf{X} \stackrel{\text { impl }}{\longrightarrow} \mathbf{Y})$, where $\mathrm{Y}$ becomes the resultant for the operations defined in $(\mathrm{X}) . \mathrm{Y}$ will be evaluated iff the operation defined in $(\mathrm{X})$ results true.

\section{4. $\mu$ Tent's Subset Properties}

$\mu$ Tent follows subset property to classify the component's as either (1) Benign, or (2) Malware. By default $\mu$ Tent source is labeled as benign. The receiver is labeled as benign iff the sender's capability $\left(C_{s}\right)$ forms a subset of the receiver's capability $\left(C_{r}\right)$, i.e. $C_{s} \subseteq C_{r}$, else the receiver is labeled as malware, i.e. $C_{s} \nsubseteq C_{r}$. By default, empty permission (i.e. when an application doesn't define any permissions in AndroidManifest.xml) is considered as a special permission. Therefore, if the receiver's has an empty capability $\left(\mathrm{C}_{\mathrm{r}}=\{\emptyset\}\right)$ and the sender has read contacts capability $\left(C_{S}=\{\right.$ READ_CONTACTS $\left.\}\right)$ then receiver's capability is not a subset of the sender's capability $\left(\mathrm{C}_{\mathrm{r}} \not \mathrm{C}_{\mathrm{s}}\right)$.

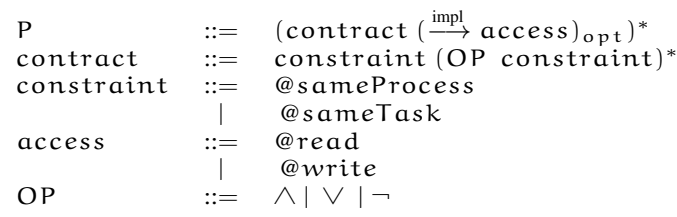

Figure 5: Arc Grammar

\section{Evaluation}

We have implemented $\mu$ Tent as a pluggable Java Library for Android (8.1+). $\mu$ Tent library contains 11 files and approximately 2,000 lines of code. We present our evaluation report for $\mu$ Tent tested using the following hardware: Android 8.1.0 (Oreo, SDK Version = 27), OnePlus 3T device with Snapdragon $821 \mathrm{CPU}$ and 6GB RAM.

\subsection{Data Set}

Our benchmark application consists of the following component's: (1) Compo ${ }^{(n, *)}$ can be either activity or broadcast receiver's or Services, where $n$ represents process/task/task-affinity to which the component belongs and $*$ represents component capability (Benign (P) or Malware (LP)).

We evaluated $\mu$ Tent's accuracy by creating a repository of 288 applications (shown in Table 3) with total 276 vulnerable paths taken from existing benchmarks [14 15] and added our own ICC applications with the following programming practices such as: data mutation, read-only, no-read, and encryption. In addition to the above benchmark, we have also tested $\mu$ Tent with the SEALANT benchmark, which consists of 135 apps with total 59 vulnerable paths.

In our test, we fixed the Arc contract (i.e. DEFAULT $\wedge @$ read) for all the examples presented in Table 2. The rule DEFAULT represents the permission subset property $(\$ 3.4$, encryption property $(\$ 3.2)$ of the $\mu$ Tent and "@read" constraint enforce the read-only property on the $\mu$ Tent within the receiver's context.

The application set from (1-6) in Table 2 consists of a total 72 apps demonstrating the scenario of sniffing between component's across different process and task. In the application set $(3,6)$, the receiver being benign performs mutation attack on $\mu$ Tent data. Though such attacks are generally assumed safe, in certain scenarios like $(\$ 2.1)$ it is required to limit mutation in order to ensure integrity of the data. Such attacks are classified as tricky ICC which $\mu$ Tent handles by using "@read" Arc contract limiting mutation of Intent data.

The application set (7-12) in Table 2 consists of 162 apps with 162 vulnerable paths. In total we have 210 vulnerable paths demonstrating the scenario of UIR and 
Table 2: Detailed Comparison

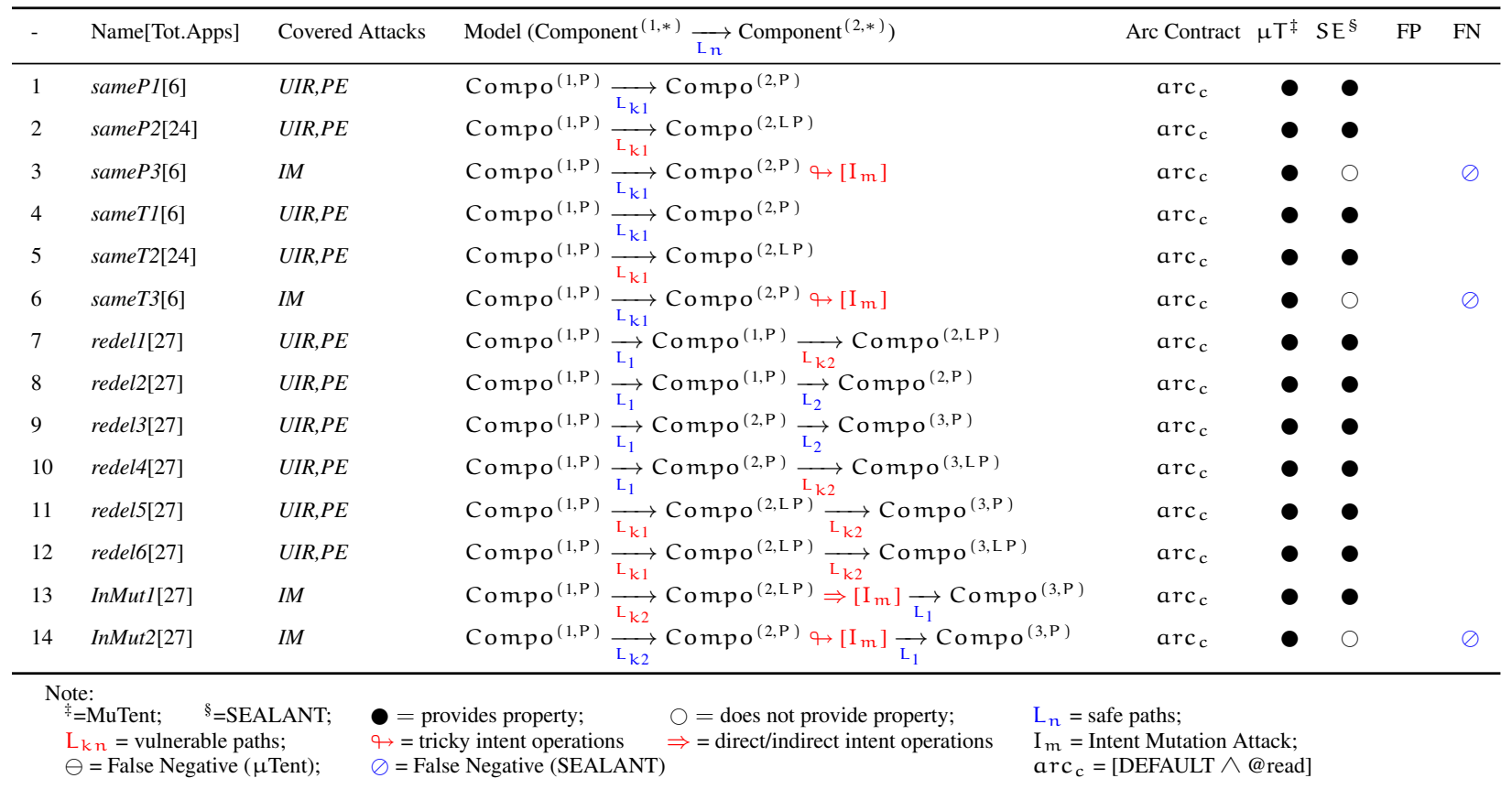

Table 3: $\mu$ Tent Evaluation.

\begin{tabular}{lcccc}
\hline Attacks & Tot.Apps & $\begin{array}{c}\text { Tot. Vulnerable } \\
\text { Paths }\end{array}$ & Blocked ( $\mu$ T) & Blocked (SE) \\
\hline UIR & 111 & 105 & $105(100 \%)$ & $105(100 \%)$ \\
PE & 111 & 105 & $105(100 \%)$ & $105(100 \%)$ \\
IM & 66 & 66 & $66(100 \%)$ & $27(40.91 \%)$ \\
Total & 288 & 276 & $276(100 \%)$ & $237(85.87 \%)$ \\
\hline
\end{tabular}

PE. $\mu$ Tent blocks all the 210 vulnerable path by using its "DEFAULT" property of validating the sender and the receiver's capability as discussed in $\$ 3.4$

Finally, we have added a collections of 54 apps to demonstrate mutation-attacks $(13,14)$. The attack scenario (13) has been filtered easily by $\mu$ Tent by validating the capability subset property, thereby the malware receiver Compo ${ }^{2, L P}$ cannot access the data. However, in attack scenario (14) the benign component is mutating the data. In order to handle such class of attacks, we used the "@read" Arc contract that specifies read-only operation to the receiver $\left(\mathrm{Compo}^{2, \mathrm{P}}\right)$, thereby limiting the receiver from modifying the data.

\subsection{Effectiveness}

Table 2 presents the detailed comparison between $\mu$ Tent and SEALANT [2]. The attack scenarios (3, 6, 14) demonstrates tricky operations performed by trusted component's, where the transit ICC path consists of only
Table 4: $\mu$ Tent Overall Impact $(\%)$.

\begin{tabular}{ccccc}
\hline Apps & $\begin{array}{c}\text { Intent } \\
\text { Creation } \\
\text { Time(ms) }\end{array}$ & $\begin{array}{c}\mu \text { Tent } \\
\text { Creation } \\
\text { Time(ms) }\end{array}$ & $\begin{array}{c}\mu \text { Tent } \\
\text { Overall } \\
\text { Dif.(ms) }\end{array}$ & $\begin{array}{c}\mu \text { Tent } \\
\text { Overall } \\
\text { Impact(\%) }\end{array}$ \\
\hline Ads Droid & 0.45 & 14.2 & 13.75 & 0.0046 \\
\cline { 2 - 5 } & 0.77 & 14.0 & 13.23 & 0.0044 \\
\hline Face Slim & 0.75 & 9.75 & 9.0 & 0.0030 \\
\hline
\end{tabular}

benign component's, which creates difficulty to restrict access based on receiver's capability and hence filtering only based on the component's capability may result in a False Negative outcome.

The other similar tools/methodology available in literature are XmanDroid [16 17] (a policy based model) and Sparta [5] (a static tool to identify the taint-flow). XmanDroid uses application level policies to block vulnerable paths. It demands the user to specify the policy defining the vulnerable ICC path. Absence of policy makes XmanDroid to allow every ICC, that is without such policy all the component's are assumed benign by default.

$\mu$ Tent differs from XmanDroid by performing permission subset validation of the receiver by default (without requiring an explicit policy specification), and protecting the payload with an ownership-based encryption model. $\mu$ Tent considers the following application-level covert channels [18] such as: 


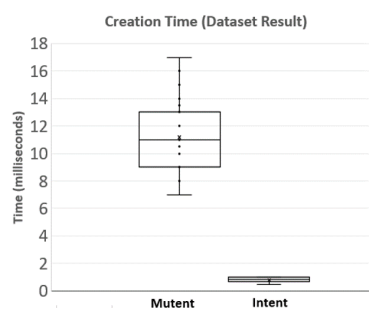

Figure 6: Overall Creation Time Overhead

broadcasts, system services, or content providers that uses Android's Intent sharing as communication medium and not any other covert channels. Also in this paper we are not considering the other operating-system level or hardware-level covert channels [19,20] such as: file system, database, $\log$, cache memory etc. $\mu$ Tent uses dynamic verification of the receiver based on receiver's capability and the Arc contract, rather than static taint analysis of source-code such as Sparta, or static apk analysis for vulnerable paths such as SEALANT.

\subsection{Performance}

Table 3 shows the overall impact of the $\mu$ Tent. We evaluate by analyzing the number of vulnerable paths present and the number of paths $\mu$ Tent's can identify as vulnerable, and can block by throwing exception. The test result is from the outcome of evaluating the $\mu$ Tent benchmark consisting of 288 total apps (\$4.1). The benchmark consists of a total 276 vulnerable paths, out-of which $\mu$ Tent can identify and block 276 paths (i.e. $100 \%$ success), and SEALANT has $85.87 \%$ accuracy by blocking 237 paths.

Additionally, we tested $\mu$ Tent with the SEALANT benchmark, which consists of 135 apps with total 59 vulnerable paths. In this test, $\mu$ Tent was able to identify and block 112 apps (with an accuracy of 82.96\%) and 48 vulnerable paths (with an accuracy of $81.36 \%$ ) that consists of redelegation attacks and Intent data access.

\subsection{Creation Time Overhead}

Since $\mu$ Tent object requires an additional dynamic owner creation along with a complex algorithm for encryption and key distribution, it becomes necessary for us to calculate the time taken to create a $\mu$ Tent so that we can calculate the overall impact $\mu$ Tent can impose on an application. Hence in this section we discuss the average creation time and its impact ratio based on our current $\mu$ Tent library implementation. We tested $\mu$ Tent by modifying three open source projects taken from F-Droid, and modified the Intent with $\mu$ Tent library (shown in Table 4). On an average $\mu$ Tent took $\sim 11.5$

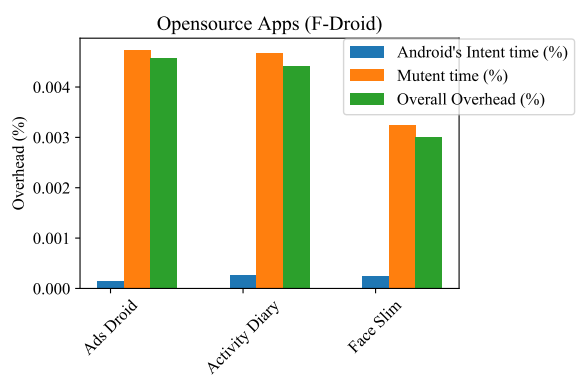

Figure 7: $\mu$ Tent Implementation Overhead

milliseconds (Figure 7) more than Android's Intent that is approximately equivalent to $0.005(\%)$ overhead in a 300,000 milliseconds of an application runtime as shown in Figure6.

We tested $\mu$ Tent using two approaches: (1) using Culebra - a record and play test environment [21] where user interactions can be recorded as python script and can be played automatically, and (2) manual testing - by giving the applications to three student volunteers from our lab and asked them to interact with the application randomly (without any pre-defined screen interaction order). Even though $\mu$ Tent's creation time is higher than Android's Intent, the overall impact percentage added by $\mu$ Tent to an applications execution is trivial (i.e. $\sim 0.005 \%$ ). Thus with this nominal impact on creation time, during our testing the users have not felt any latency in the screen response and screen navigation.

\section{Related Work}

Detecting data-leaks and malicious component's during Inter Component Communication (ICC) have already been explored for source code and DEX file. These approaches combines static analysis and runtime policies at the OS level (i.e., requires modifying the framework or kernel code). However, $\mu$ Tent is designed and implemented as a pluggable Java Intent library to filter the data-leaks and malicious component's during ICC. Encrypting ICC payload is generally treated as a OS layer functionality, which $\mu$ Tent introduce as Intent property.

Static Analysis: SEALANT [2] provides a technique that combines static analysis of app code infering vulnerable communication channels and runtime monitoring of inter-app communication through those channels to prevent attacks. SALMA [4], presents self-protecting Android system using a Multiple-Domain-Matrix (a knowledge base), that will be updated incrementally and thereby enabling incremental analyzes upon changes to the system. FlowDroid [22] a static taint-analysis reduces false alarm 
by detecting the data-leaks based on the context, flow, field and object-sensitivity. Amandroid [23] statically detects inter-component control and data flows among multiple component's from either the same or different apps. Since $\mu$ Tent's objective is to prevent Intent-based attacks by creating an Intent library we do not perform static analysis like the above models; however $\mu$ Tent's dynamic mechanism for adding contract to the Intent helps to enable data integrity and to control accessibility and visibility of the data. ComDroid [24] analyzes the DEX files to detect Intent-based attacks such as Intent spoofing, UIR and Intent vulnerabilities by performing flow-sensitive and intra-procedural static analysis. CHEX [25] approaches the detection problems such as leak of private data or component vulnerability as data-flow problem that helps to identify the potential risk from the existence of flow pattern. AnFlo [26 proposes a mechanism to detect anomalous sensitive data flows in Android apps by grouping trusted apps based on the categories identified from the apps functional descriptions. TERMINATOR [27] analyzes the permission misuse by considering the order of events that require the time of an event and the security vulnerability in the Android's custom permission model. In $\mu$ Tent every ICC call must have a valid capability to access the Intent data, thereby the flow graph varies in par with the users permission decision for that application.

Policies \& Filters: Kirin [28] detects the malicious behavior by certifying an app using kirin security rules at install time. DREBIN [29] proposed a method for detection of Android malware by uses dynamic inferring of security patterns rather than manually crafted patterns [28]. TaintDroid [30], a data-leak detection tool, suggested a fine-grained tracking of private information and how it is actually used. $\mu$ Tent's context-based privacy using dynamic contracts was inspired from the above works; during ICC every $\mu$ Tent is controlled by a unique Trusted Computing Base (TCB) that is created per object. Permission re-delegation introduces a risk when an application with permissions performs a privileged task for an application without permissions. The permission re-delegation paper [3] proposed IPC inspection at OS level, that reduces a deputy's (application with permissions) privileges after receiving communication from a malware application (app without privileges). Consequently, the Android's permission system can deny a privileged API call from the deputy if any application in the chain of influence lacks the appropriate permission(s). DroidCap [31] uses Binder handles as capability tokens by associating Android's permissions to the handle. DroidCap facilitates privilege separation between app's component's through compartmentalization of component's into a logical app with a subset of privileges. Maxoid [32] confines the delegates from leaking secrets by creating different views of the initiator's state. The delegates may update initiator private state or public state which the initiator can selectively commit or discard to prevent unwanted modifications by delegates. This may not be directly applied to Intent data protection, since Intent communication creates a uni-directional data distribution, where the shared Intent data cannot be controlled by the initiator unless the data is represented as a URI. Aquifer [33] defines UI workflow policies where each participating application define its export list, a required list, and a workflow filter. Aquifier tracks the workflow policies to control the interactions across different applications who are participating to complete the task. Similarly, in $\mu$ Tent we compare the capability of an application before allowing the receiver to access the Intent data, since Intent's are initiated within the receiver's context, we provide an additional encryption mechanism to limit the receiver from accessing the data through reflection.

Encryption \& Key Distribution: Srivatsa, et al. [6] provided routable attribute protection using content-based routing where the publisher uses event key $K(e)$ to encrypt, and subscriber with filter (f) uses key $K(f)$ to decrypt, where $K(f)$ is capability of a subscriber and hence treated equally. On the other hand, the $\mu$ Tent handles subscriber's based on the capability and the Arc contract, rather based on subscription.

\section{Conclusion}

We have presented $\mu$ Tent, the secure and pluggable Android Intent Library written in Java, to securely exchange intent data across component's during ICC. $\mu$ Tent is the first holistic model to provide the dynamic control of intents inside the subscriber's context. It differs from the earlier model in terms of providing dynamic encryption, ownership based key distribution, and verification of the receiver's context using user defined security contracts and receiver's capability. Thereby with $\mu$ Tent, we enable application component's to sensibly retain the control of their intent data after it has been shared with the receiver during ICC.

\section{References}

[1] "Vulnerability Details : CVE-2018-9489.." https:// WWW.cvedetails.com/cve/CVE-2018-9489 
[2] Y. K. Lee, J. andg Bang, G. Safi, A. Shahbazian, Y. Zhao, and N. Medvidovic., "A SEALANT for Inter-app Security Holes in Android.," in Proceedings of the 39th ICSE, pp. 312-323, 2017.

[3] F. A.P., W. H.J., M. A., H. S., and C. E., "Permission Re-Delegation: Attacks and Defenses.," in USENIX Symposium, p. 22, 2011.

[4] M. Hammad, J. Garcia, and S. Malek, "Self-Protection of Android Systems from Inter-Component Communication Attacks.," in 33rd ACM/IEEE ASE'18, p. 726-737, 2018.

[5] P. Barros, R. Just, S. Millstein, P. Vines, W. Dietl, M. d'Amorim, , and M. D. Ernst., "Static analysis of implicit control flow: Resolving Java reflection and Android intents.," in Proceedings of 30th ASE'15, p. 669-679, 2015

[6] M. Srivatsa, L. Liu, and A. Iyengar., "EventGuard: A System Architecture for Securing Publish-Subscribe Networks.," in ACM TOCS, vol. 29, 2011.

[7] A. Feizollah, N. B. Anuar, R. Salleh, G. Suarez-Tangil, and S. Furnell., "AndroDialysis: Analysis of Android Intent Effectiveness in Malware Detection.," in Elsevier Computers \& Security, pp. 121-134, 2017.

[8] M. Heiderich, J. Schwenk, T. Frosch, J. Magazinius, and E. Z. Yang., "mXSS attacks: attacking well-secured web-applications by using innerHTML mutations.," in ACM SIGSAC CCS'13, pp. 777-788, 2013.

[9] C. M. Stone, T. Chothia, and F. D. Garcia., "Spinner: Semi-Automatic Detection of Pinning without Hostname Verification.," in 33rd ACSAC'17, pp. 176-188, 2017.

[10] "Man-in-the-Middle Flaw in Major Banking, VPN Apps Exposes Millions.." https : / /www. darkreading . $\mathrm{com} / \mathrm{mobile/man-in-the-middle-flaw-in}$ -major-banking-vpn-apps-exposes-mill ions/d/d-id/1330586

[11] A. Nadkarni, B. Andow, W. Enck, and S. Jha, "Practical DIFC Enforcement on Android," in 25th USENIX'16, pp. 1119-1136, 2016.

[12] D. Clarke and S. Drossopoulou., "Ownership, encapsulation and the disjointness of type and effect.,' in 17th ACM OOPSLA'02, pp. 292-310, 2002.

[13] L. Burdy, Y. Cheon, D. R. Cok, M. D. Ernst, J. R. Kiniry, G. T.Leavens, K. R. M. Leino, and E. Poll, "An Overview of JML Tools and Applications.," in FMICS'03, pp. 75-91, 2003.

[14] "ICC-Bench: Benchmark apps for static analyzing inter-component data leakage problem of Android apps.." https://github.com/fgwei/ICC-B ench

[15] "DroidBench 2.0: A micro-benchmark suite to assess the stability of taint-analysis tools for Android.." https: //github.com/secure-software-enginee ring/DroidBench

[16] S. Bugiel, L. Davi, A. Dmitrienko, T. Fischer, and A.-R. Sadeghi., "XManDroid: A New Android Evolution to Mitigate Privilege Escalation Attacks.," in Technische Universität Darmstadt, TR-2011-04, 2011.

[17] S. Bugiel, L. Davi, A. Dmitrienko, T. Fischer, A.-R. Sadeghi, and B. Shastry., "Towards Taming Privilege-Escalation Attacks on Android.," in NDSS Symposium, 2012.
[18] C. Marforio, H. Ritzdorf, A. Francillon, and S. Capkun., "Analysis of the communication between colluding applications on modern smartphones.," in Proceedings of the 28th ACSAC'12, pp. 51-60, 2012.

[19] H. Okhravi, S. Bak, and S. T. King., "Design, Implementation and Evaluation of Covert Channel Attacks.," in IEEE International Conference on Technologies for Homeland Security (HST), Waltham, MA, pp. 481-487, 2010.

[20] H. Ritzdorf, "Analyzing Covert Channels on Mobile Devices.," in Master Thesis. ETH Zürich, Department of Computer Science, 2012.

[21] "Culebra: Generating Ready-to-Execute Scripts for Black box testing.." https://github.com/dtm ilano/AndroidViewClient/wiki/culebra

[22] S. Arzt, S. Rasthofer, C. Fritz, E. Bodden, A. Bartel, J. Klein, Y. L. Traon, D. Octeau, and P. McDaniel., "FlowDroid: precise context, flow, field, object-sensitive and lifecycle-aware taint analysis for Android apps.," in PLDI'14, pp. 259-269, 2014.

[23] F. Wei, S. R. X. O., and Robby., "Amandroid: A Precise and General Inter-Component Data Flow Analysis Framework for Security Vetting of Android Apps.," in Proceedings of ACM CCS'14, pp. 1329-1341, 2014.

[24] E. Chin, A. Porter, F. K. Greenwood, and D. Wagner., "Analyzing Inter-Application Communication in Android." in Proceedings of the 9th MobiSys, pp. 239-252, 2011.

[25] L. Lu, Z. Li, Z. Wu, W. Lee, and G. Jiang, "CHEX: statically vetting Android apps for component hijacking vulnerabilities.," in Proceedings of the ACM CCS, p. 229-240, 2018.

[26] B. F. Demissie, M. Ceccato, and L. K. Shar., "AnFlo: detecting anomalous sensitive information Flows in Android apps.," in Proceedings of MOBILESoft, p. 24-34, 2018.

[27] A. Sadeghi, R. Jabbarvand, N. Ghorbani, H. Bagheri, and S. Malek., "A temporal permission analysis and enforcement framework for Android.," in Proceedings of the 40th ICSE, pp. 846-857, 2018.

[28] W. Enck, M. Ongtang, and P. McDaniel, "On Lightweight Mobile Phone Application Certification.," in Proceedings of the ACM CCS, p. 235-245, 2009.

[29] D. Arp, M. Spreitzenbarth, M. Hubner, H. Gascon, and K. Rieck, "DREBIN: Effective and Explainable Detection of Android Malware in Your Pocket.," in NDSS'14, pp. 23-26, 2014.

[30] W. Enck, P. Gilbert, B.-G. Chun, L. P. Cox, J. Jung, P. Mcdaniel, , and A. N. Sheth., "TaintDroid: An information-flow tracking system for realtime privacy monitoring on smartphones.," in 9th USENIX (OSDI'10), pp. 393-407, 2010.

[31] A. Dawoud and S. Bugiel., "DroidCap: OS Support for Capability-based Permissions in Android.," in NDSS Symposium, 2019.

[32] Y. Xu and E. Witchel., "Maxoid: Transparently confining mobile applications with custom views of state," in EuroSys'15, pp. 1-16, 2015.

[33] L. Jia, J. Aljuraidan, E. Fragkaki, L. Bauer, M. Stroucken, K. Fukushima, S. Kiyomoto, and Y. Miyake., "Run-time enforcement of information-flow properties on Android," in European Symposium on Research in Computer Security, pp. 775-792, 2013. 